\title{
MICROSCOPIA ÓPTICA AUTOMÁTICA COM PROCESSAMENTO DE IMAGENS APLICADA A ANÁLISE DE MINÉRIO DE FERRO, SÍNTER E PELOTAS*
}

\author{
Alei Leite Alcantara Domingues ${ }^{1}$ \\ Valdirene Gonzaga de Resende ${ }^{2}$ \\ Ana Paula Calazans Gomes ${ }^{3}$ \\ Etevani Andrade Gomes ${ }^{4}$ \\ Aloísio Antônio Melo Borges 5
}

\begin{abstract}
Resumo
A microscopia óptica é uma ferramenta importante para o entendimento dos processos envolvidos na produção do minério de ferro e seus aglomerados. Nos laboratórios em que esse tipo de análise é feita, tem-se geralmente um microscópio de luz refletida e um operador treinado que leva diversas horas para concluir uma única amostra. Nesse trabalho serão mostrados exemplos de resultados de minérios de ferro, sínter e pelotas obtidos em um sistema de microscopia óptica automática com análise de imagens. No estudo do minério de ferro foram determinados a composição mineralógica quantitativa, morfologia das partículas e tamanho dos cristais. Na microscopia de sínter foram determinados a quantificação de fases, porosidade, textura, recristalização de hematita e morfologia das ferritas. Nas pelotas produzidas com minério de ferro foram feitas análises de quantificação de fases, distribuição e quantificação dos poros e evolução microestrutural dos grãos. A implantação de um sistema de microscopia óptica automática com análise de imagens permite que análises sejam feitas em menor tempo, com maior reprodutibilidade possibilitando que a técnica seja implantada em áreas de produção que exijam respostas mais rápidas.
\end{abstract}

Palavras-chave: Análise de Imagens; Microscopia Óptica; Microscopia de Minério de Ferro; Microscopia de Sínter; Microscopia de Pelota.

\section{AUTOMATED OPTICAL MICROSCOPY WITH IMAGE PROCESSING APPLIED TO IRON ORE, SINTER AND PELLET ANALYSIS}

\begin{abstract}
Optical microscopy is an important tool for understanding the processes involved in the production of iron ore and its agglomerates. In laboratories, in which this type of analysis is performed, there is usually a reflected light microscope and a qualified operator that takes several hours to complete the analysis of a single sample. In this work results of iron ore, sinter and pellets obtained in an automatic optical microscopy system with image analysis is shown. Quantitative mineralogical composition, particle morphology and crystal size were determined for the iron ore. For the sinter microscopy analysis, phase quantification, porosity, texture, hematite recrystallization and ferrite morphology were determined. In the pellets produced with iron ore, phase quantification, distribution and quantification of the pores, and microstructural evolution of the grains were performed. The system of automated optical microscopy with image analysis allows analyzes to be performed in a shorter time and with higher reproducibility, allowing the technique to be implemented in production areas that require faster responses.
\end{abstract}


Keywords: Image Analysis; Optical Microscopy; Microscopy of Iron Ore; Microscopy of Sínter; Microscopy of Pellet.

1 Engenheiro Geólogo, Centro de Tecnologia de Ferrosos, Vale S.A., Nova Lima, MG, Brasil.

2 Química Industrial, Centro de Tecnologia de Ferrosos, Vale S.A., Nova Lima, MG, Brasil.

3 Técnico Mineração, Centro de Tecnologia de Ferrosos, Vale S.A., Nova Lima, MG, Brasil.

4 Técnico Mineração, Centro de Tecnologia de Ferrosos, Vale S.A., Nova Lima, MG, Brasil.

5 Engenheiro de Minas, Centro de Tecnologia de Ferrosos, Vale S.A., Nova Lima, MG, Brasil. 


\section{INTRODUÇÃO}

A microscopia óptica tem sido uma importante ferramenta de análise para a indústria mínero-metalúrgica desde tempos mais remotos. Na mineração a microscopia óptica é amplamente empregada na determinação da composição mineralógica dos minérios, morfologia dos minerais e liberação dos grãos, enquanto na indústria metalúrgica é utilizada para análise dos aglomerados, combustíveis e na avaliação da microestrutura dos aços. A utilização da microscopia automática com análise de imagens, permite a otimização de alguns desses processos, tornando as análises mais rápidas e precisas, além de aumentar a quantidade de características que podem ser determinadas [1].

Nesse trabalho serão descritas as características de um sistema de microscopia automática com análise de imagens e mostrados exemplos da utilização em alguns dos produtos da cadeia mínero-metalúrgica: minério de ferro, sínter e pelotas. Em um projeto desenvolvido pela Vale com apoio da Pontifícia Universidade Católica do Rio (PUC-Rio), novos métodos baseados em microscopia automática e análise de imagens foram desenvolvidos, automatizando algumas etapas de caracterização [2], com a medição de frações de fase e porosidade. Uma das vantagens desse sistema é a possibilidade de aquisição de mosaicos que cobrem áreas muito maiores que em um único campo [3]. As análises são realizadas no Centro de Tecnologia de Ferrosos (CTF) em Nova Lima - MG em trabalhos que se inserem no contexto do conhecimento geometalúrgico. Nessa forma de abordagem, todas as etapas da cadeia mínero-metalúrgica, desde a formação geológica das jazidas até a produção do aço estão relacionadas, uma vez que as características de cada etapa afetarão os processos e os resultados da etapa posterior (Figura 1). A microscopia óptica pode ser utilizada em todas as etapas dessa cadeia produtiva.

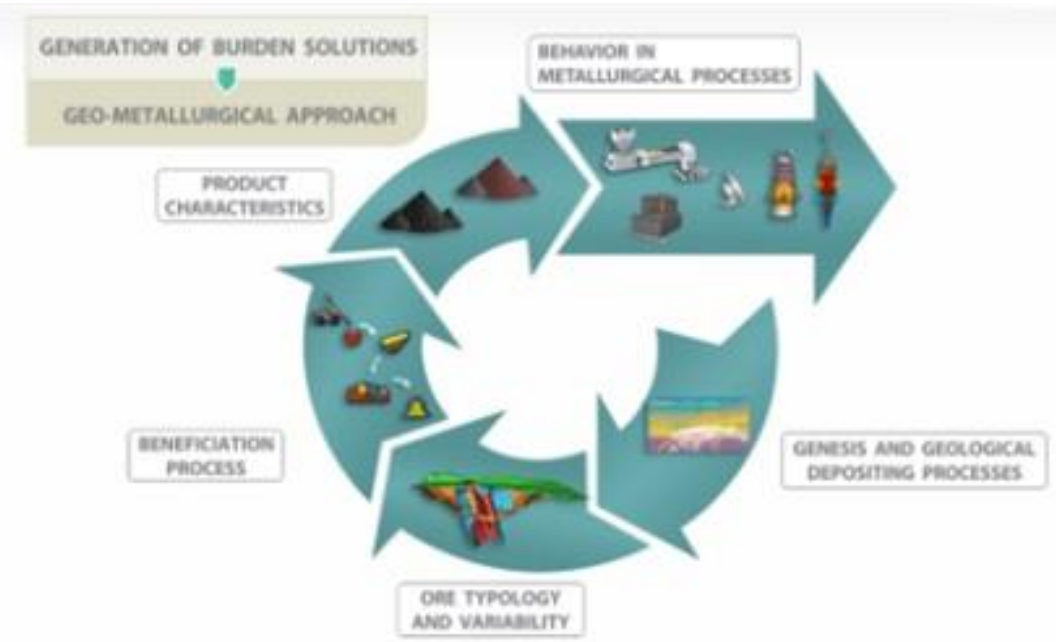

Figura 1. Esquema da abordagem geometalúrgica em que as etapas são dependentes umas das outras na cadeia mínero-metalúrgica [1].

\section{MATERIAIS E MÉTODOS}

Os recursos necessários em um sistema de microscopia automática com análise de imagens são, de uma forma geral, equipamentos de preparação de seções polidas, microscópios automatizados com câmeras de alta resolução, computadores com processadores de alta velocidade e servidores para armazenamento das imagens (Figura 2). A preparação de seções polidas que geralmente consiste em embutir, 
lixar e polir as amostras, adquire um papel muito importante em um sistema de análise de imagens, uma vez que qualquer imperfeição na superfície pode induzir as rotinas computacionais ao erro.

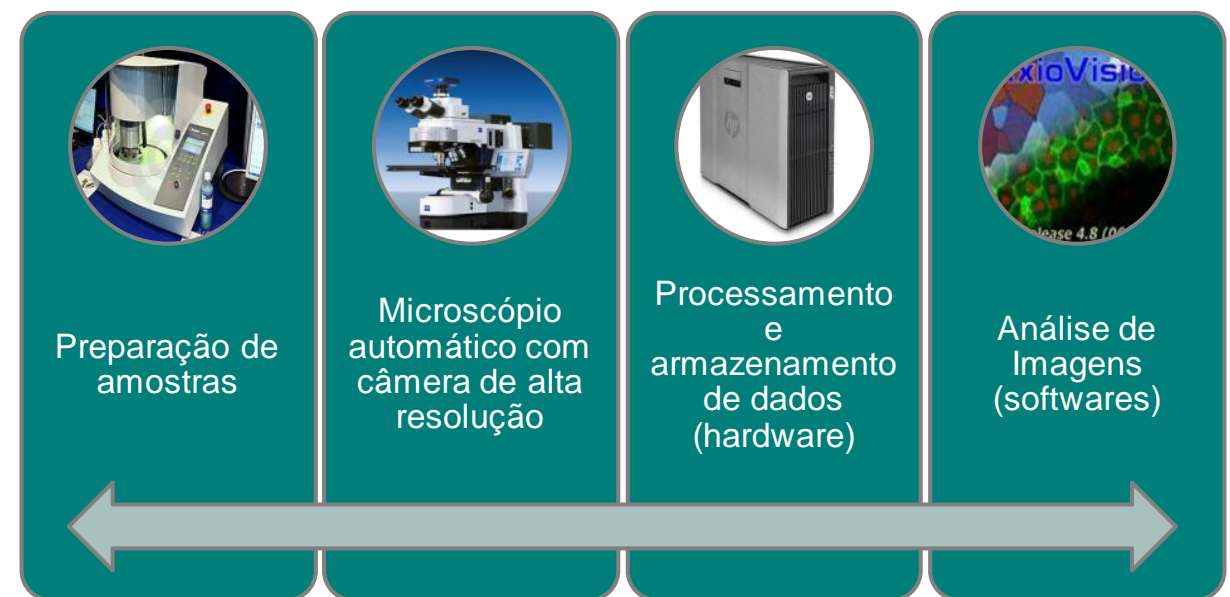

Figura 2. Recursos necessários para um sistema de microscopia automática com análise de imagens.

Nesse trabalho, foram utilizados equipamentos de preparação automáticos e de precisão como a politriz Struers Tegramin 30 e a máquina de corte Accutom 50 . Os microscópios são da marca Carl Zeiss modelo Axiolmager Z2m, com platina automática e câmera de alta resolução Axiocam MRc. Os microscópios são controlados por computadores com sistema operacional Windows e pelo software AxioVision 4.9.1, onde são obtidas as imagens e realizadas algumas operações básicas. Há um outro computador equipado com sistema operacional Linux (Debian), de alta performance com processadores configurados em esquema multicore, que é responsável por operações mais complexas (Figura 3).

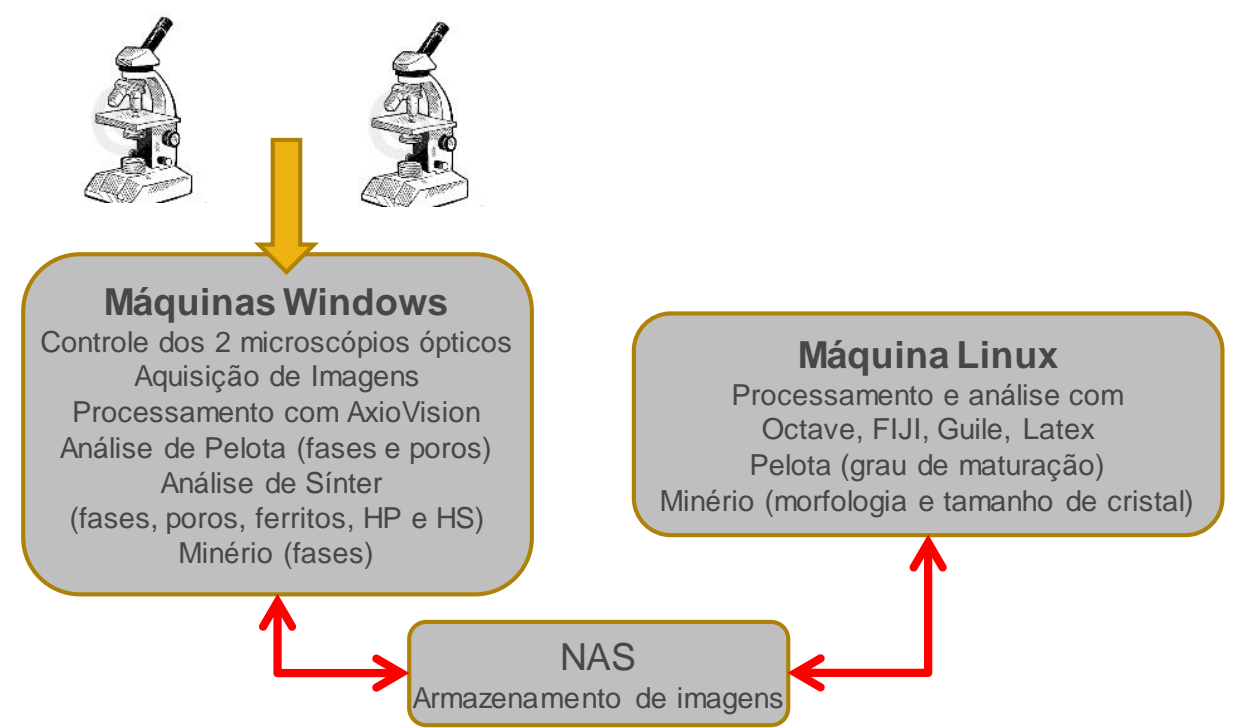

Figura 3. Esquema de montagem do sistema de análise de imagens.

De acordo com a granulometria do minério de ferro, pode ser necessário realizar uma adequação granulométrica antes do embutimento. Nesse trabalho, para análise de mineralogia quantitativa, todo o minério foi cominuído até que estivesse abaixo de $0,250 \mathrm{~mm}$. No caso do sínter, foram coletados fragmentos entre $10 \mathrm{~mm}$ e $16 \mathrm{~mm}$, enquanto as pelotas foram analisadas cortadas ao meio. 


\section{RESULTADOS E DISCUSSÃO}

\subsection{Análise de minério de ferro}

O estudo do minério de ferro que geralmente é feito pela exaustiva análise por contagem de pontos (ou de partículas) visa determinar a composição mineralógica quantitativa, morfologia das partículas e liberação dos grãos. Essas características são importantes para a determinação da qualidade dos produtos e pelo comportamento nos processos de beneficiamento, aglomeração e redução. As rotinas desenvolvidas para minério de ferro utilizam microscópios de luz refletida com luz polarizada e nicóis cruzados. Enquanto a análise tradicional pode exigir até três horas de um operador treinado contando campos na ocular do microscópio, na análise de imagens os mesmos resultados podem ser gerados em cerca de vinte minutos. No CTF há dois módulos de análise de minério de ferro: básico e avançado O módulo básico é processado no software AxioVision e consiste em determinar as fases a partir do seu comportamento óptico de reflectância, que possuem comportamento distinto no histograma da imagem (Figura 4). As imagens são georreferenciadas pela platina automática do microscópio e a quantidade de cada fase é dada pela área que ocupa.
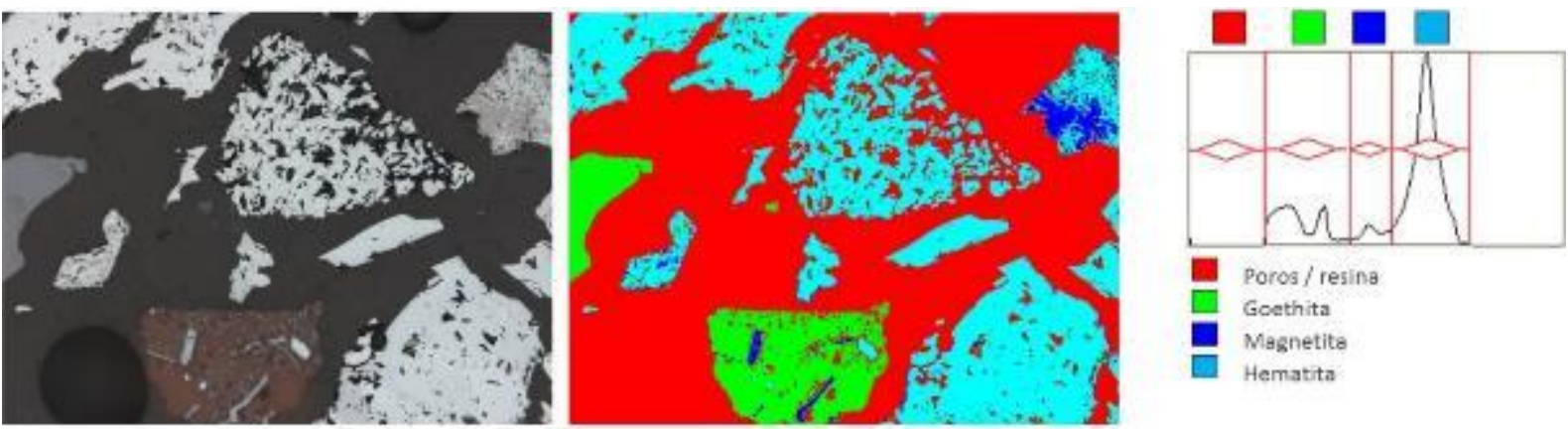

Figura 4. (A) Típica imagem de minério de ferro em campo claro (B) mesma imagem com falsas cores após a segmentação do histograma.

O modulo avançado [4-7] é processado em ambiente Linux e é dedicado a análise das hematitas, com a distinção dos tipos morfológicos e tamanhos de cristal. São obtidas imagens em campo claro e polarizada utilizando o polarizador circular (CPOL). Ele foi programado em duas etapas sequenciais:

- A primeira etapa chamada de Método Sintético, separa as hematitas em dois domínios: porosas e compactas. As hematitas porosas serão classificadas como hematita microcristalina ou martita;

- A segunda etapa, designada Método Analítico, analisa as hematitas compactas e as classifica como hematitas lamelares, granulares ou lobulares, além de medir cada cristal individualmente.

Cada partícula é analisada a partir de duas imagens obtidas em campo claro (Figura 5a) e em luz polarizada (com polarizador circular CPOL) (Figura 5b). Em seguida é realizado um comando computacional que alinha as duas imagens para que sejam delineados os cristais daquela partícula (Figura 5c). Na última etapa ocorre a identificação da morfologia do mineral (Figura 5d). 

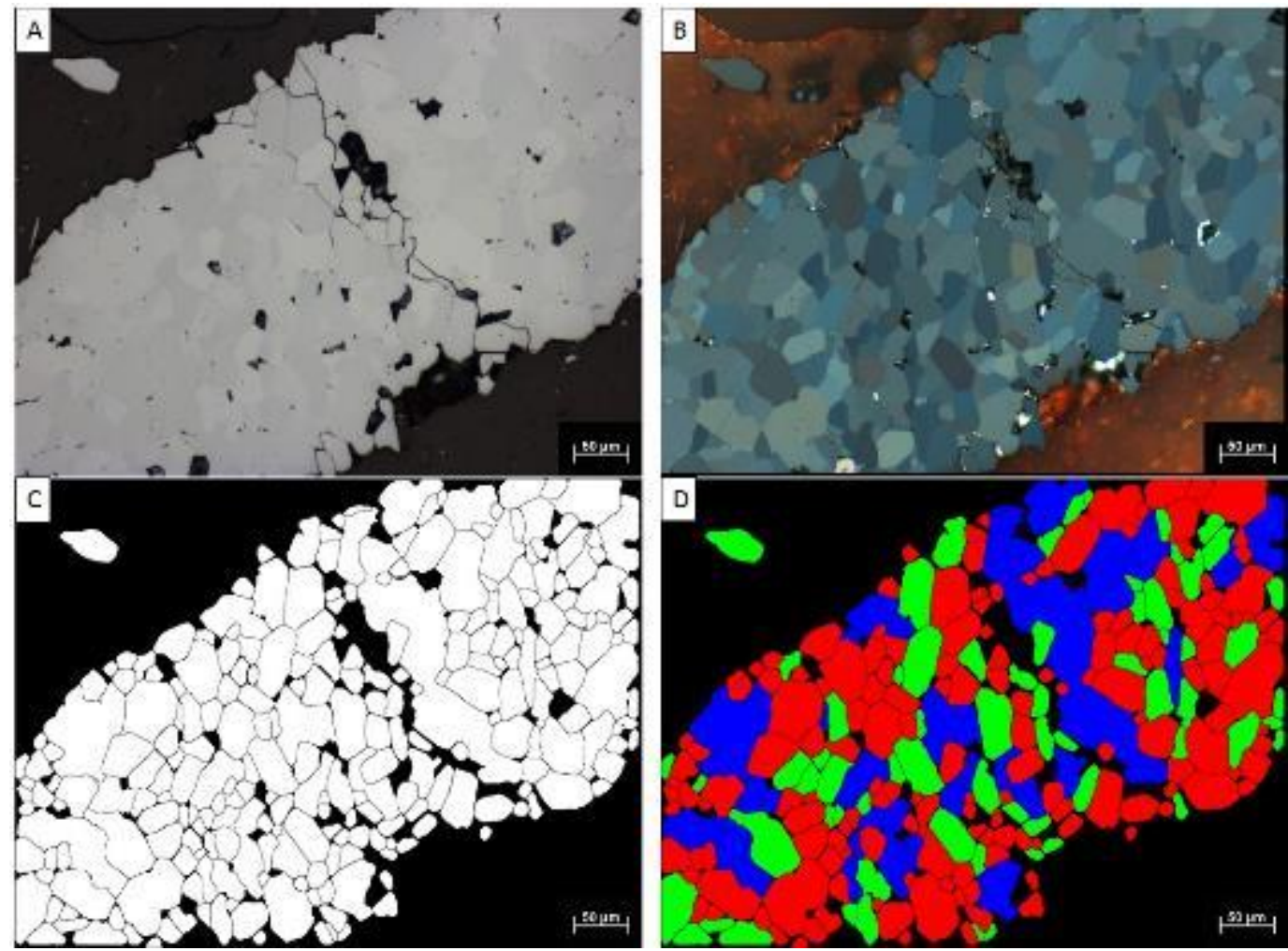

Figura 5. Hematita policristalina (A) Imagem em campo claro, (B) imagem CPOL, (C) reconhecimento dos cristais, (D) reconhecimento morfológico.

\subsection{Análise de sínter}

A microscopia de sínter é bastante útil na determinação de características que podem ser importantes para a qualidade física e desempenho de redução nos altosfornos: composição de fases, porosidade, textura, recristalização de hematita e morfologia das ferritas [8]. As análises são feitas com frequência para verificar o impacto da qualidade das matérias-primas na microestrutura dos aglomerados.

Os fragmentos de sínter foram analisados a partir de um mosaico de aproximadamente 90 imagens em campo claro, obtidas com a objetiva de $5 x$ (Figura 6a). Em seguida, as fases foram determinadas a partir do histograma da imagem (Figura 6b). Por microscopia óptica é possível determinar hematita, magnetita, ferritas e escórias (silicatos em geral). Há certa limitação na distinção entre diferentes tipos de ferritas e silicatos, por apresentarem o mesmo comportamento óptico ao microscópio de luz refletida. Caso seja necessário fazer essa distinção, recomenda-se a utilização do microscópio eletrônico de varredura.

Em seguida foi realizada a quantificação dos poros (Figura 7a). Como os poros são preenchidos com resina, a rotina computacional utiliza o histograma da imagem para quantificar apenas os poros que estão no interior do fragmento. Além de quantificar a área ocupada pelos poros, é possível realizar uma série de medidas e obter uma estatística completa de porosidade. Por fim, foi feita de forma automática uma identificação de texturas de hematita (Figura 7b). As hematitas primárias são aquelas originais do minério, enquanto as hematitas secundárias sofreram algum tipo de modificação durante o processo de sinterização que é realizado a altas temperaturas. 

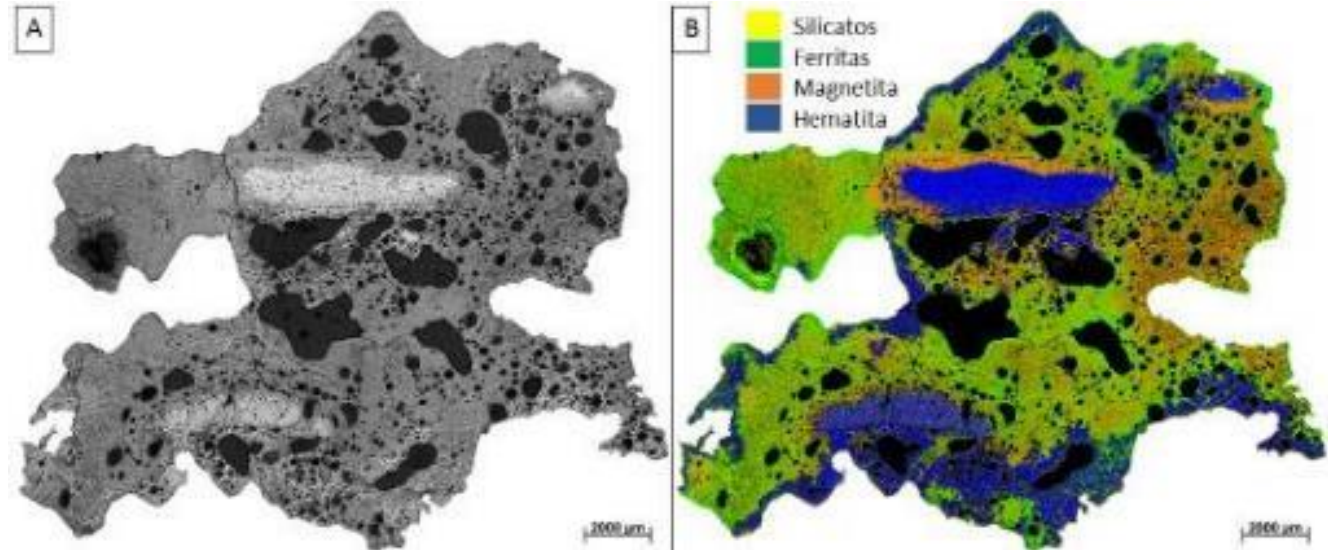

Figura 6. Imagens de mosaico. (A) Fragmento de sinter em imagem de campo claro; (B) imagem colorida com a segmentação das fases.
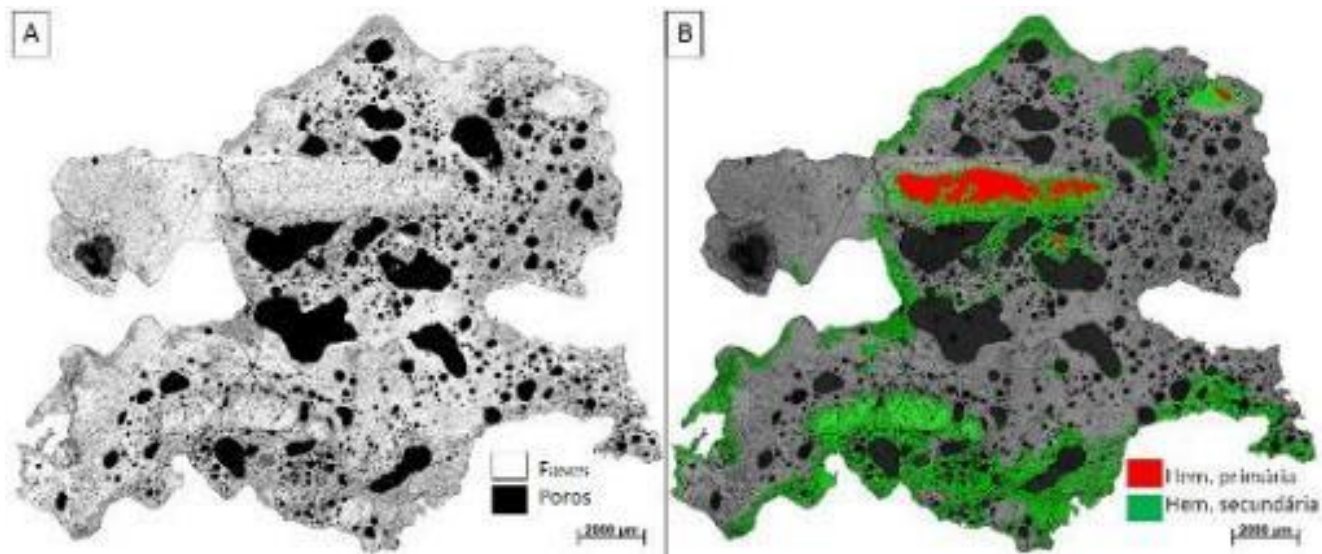

Figura 7. Imagens de mosaic. (A) Medição da fração de área da porosidade; (B) imagem colorida segmentada com os tipos texturais de hematita.

Uma outra análise possível de ser feita é a identificação da morfologia das calcioferritas, que podem ser aciculares ou colunares. Essa informação é importante uma vez que as calcioferritas aciculares contribuem para a melhoria da resistência física dos sínteres. Nesse caso, o sínter é analisado britado e não em fragmentos. Ao final da análise, o sistema gera automaticamente um relatório completo em extensão .pdf, pronto para ser enviado ao solicitante (Figura 8). 


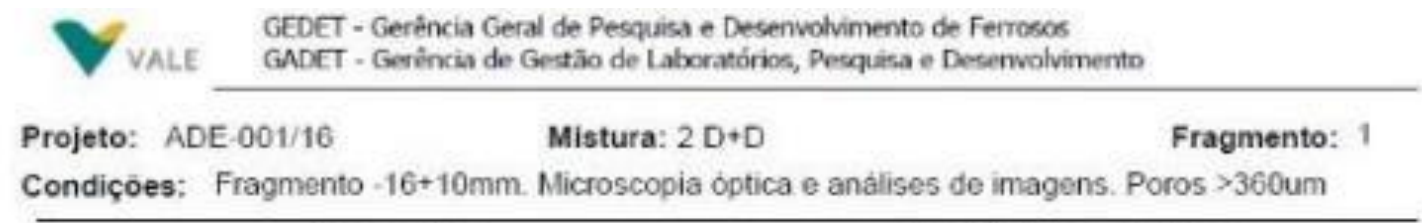
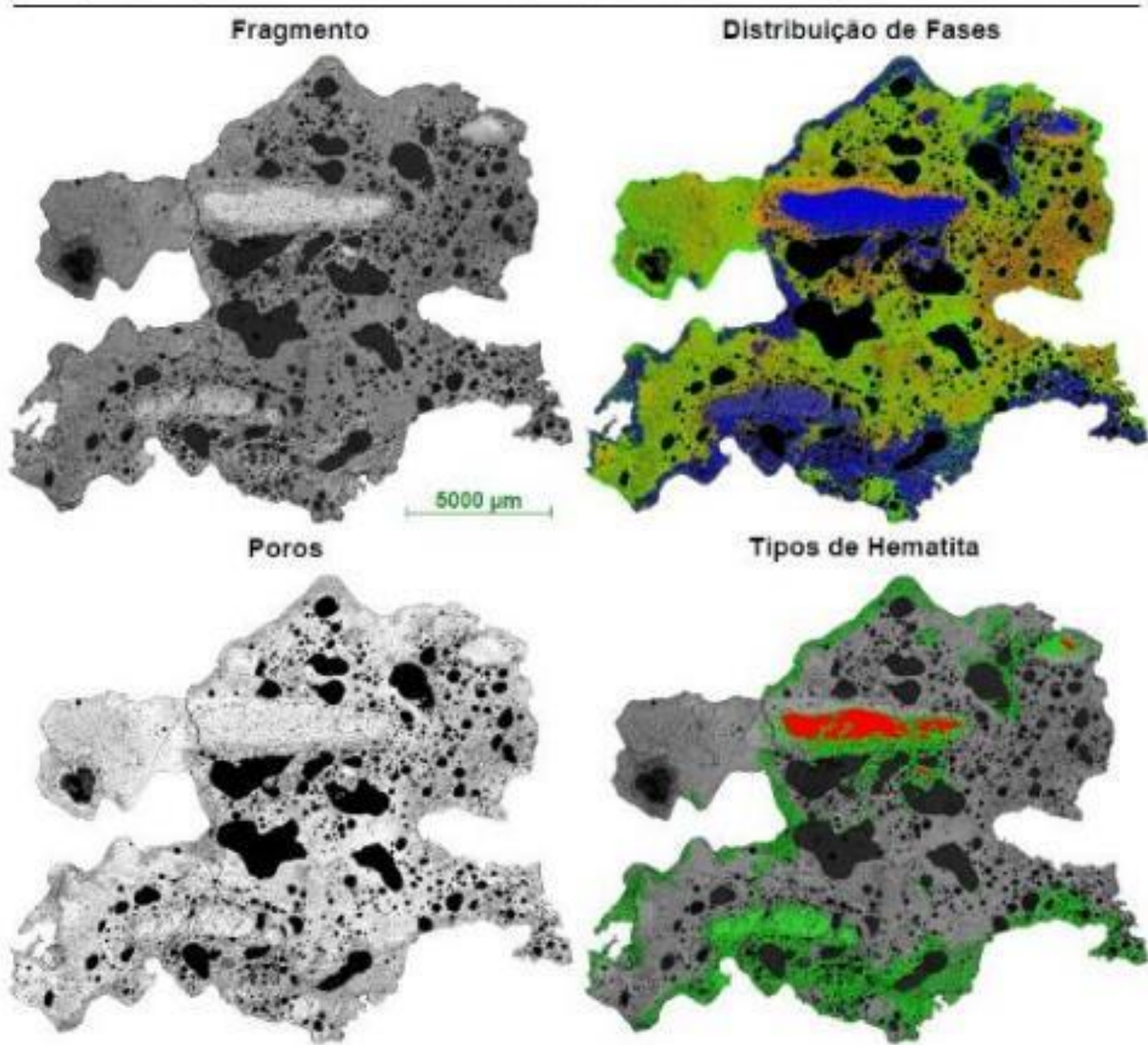

Tipos de Hematita
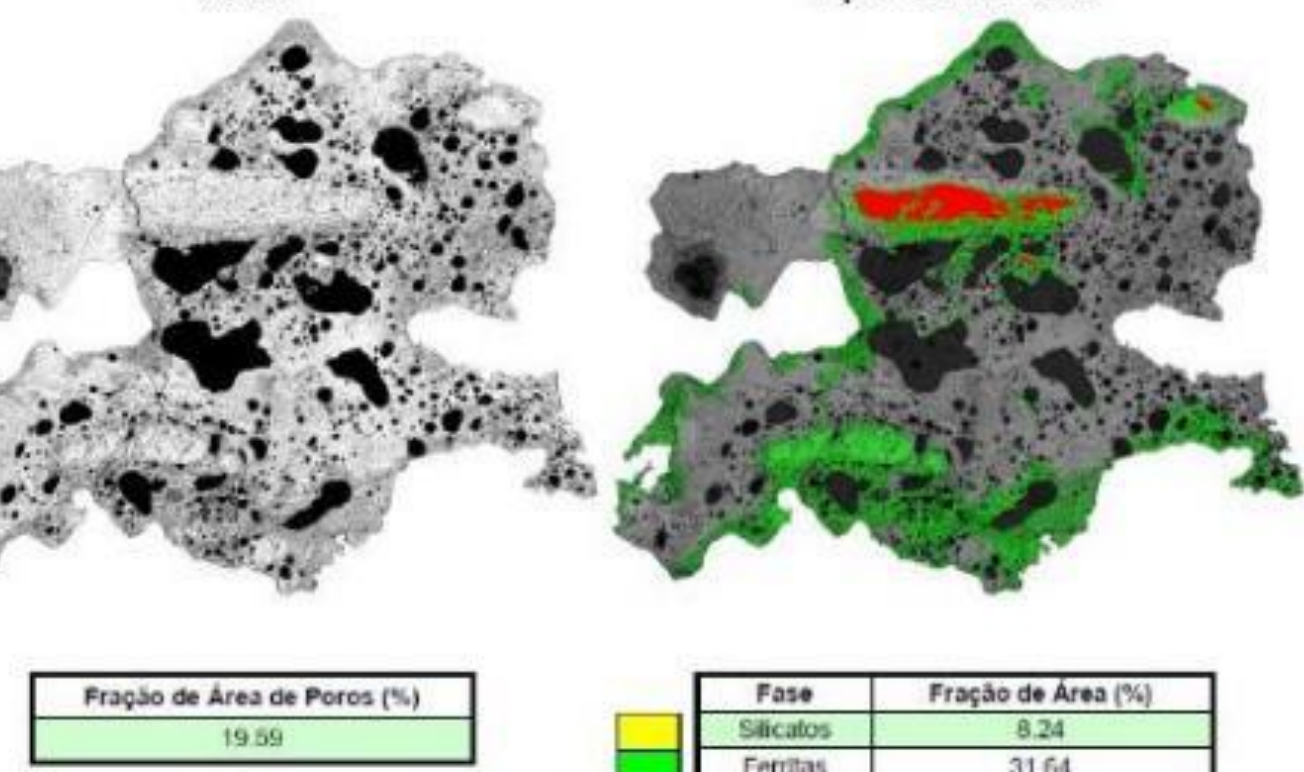

Tamanho de Poros

\begin{tabular}{|c|c|}
\hline Descricäo & DiamMix [um] \\
\hline Minimo & 423.68 \\
\hline Máximo & 14223.98 \\
\hline Médla & 1247.53 \\
\hline Desvio & 1821.14 \\
\hline 10. Percenti & 51931 \\
\hline 90-Percenti & 2240.67 \\
\hline
\end{tabular}

\begin{tabular}{|c|c|}
\hline Fase & Fraçăo de Área (\%) \\
\hline Slicatos & 8.24 \\
\hline Ferritas & 31.64 \\
\hline Magnetita & 39.96 \\
\hline Hemattia & 20.15 \\
\hline
\end{tabular}

Na fraça de Slicato estäo induldos SFCA. quartoo, olmnak, serpentiontos ef findertes najo reagudis. Na fracio de Mogrentta ustä incluida tambert a Ma-ferite.

\begin{tabular}{|c|c|}
\hline TipoHematita & $\%$ \\
\hline Primairia & 16.99 \\
\hline Secundária & 83.01 \\
\hline
\end{tabular}

Figura 8. Exemplo de um relatório automático gerado após a execução da rotina de análise de sínter.

\subsection{Análise de pelota}

Nas pelotas produzidas com minério de ferro foram feitas análises de composição de fases, distribuição e quantificação dos poros e evolução microestrutural dos grãos 
[9]. Todas essas características têm implicações na resistência física das pelotas e no seu comportamento sob atmosfera redutora. Primeiramente, foi obtido um mosaico da pelota inteira com objetiva de 10x composto por cerca de 150 imagens (Figura 9a). Em seguida foi analisada a concentração e distribuição dos poros no interior da pelota (Figura 9b).
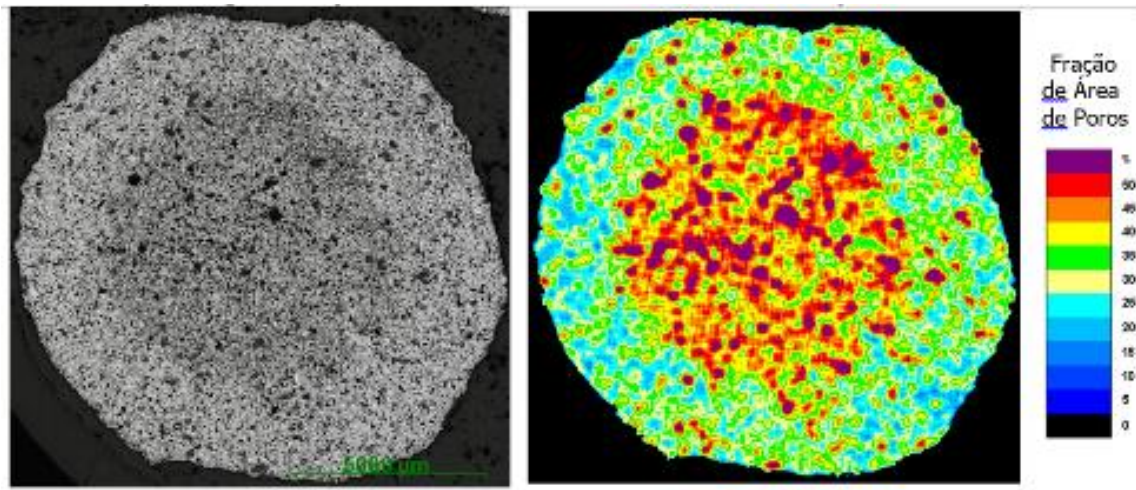

Figura 9. Imagens de mosaico. (A) Seção completa de uma pelota de minério de ferro, (B) distribuição e concentração dos poros.

Na próxima etapa obteve-se um mosaico diametral da pelota, com objetiva de 20x e cerca de 60 imagens, onde são analisados a casca, manto externo, manto interno e núcleo da pelota (Figura 10). Nessa imagem são determinados a evolução microestrutural dos grãos e a quantificação das fases.
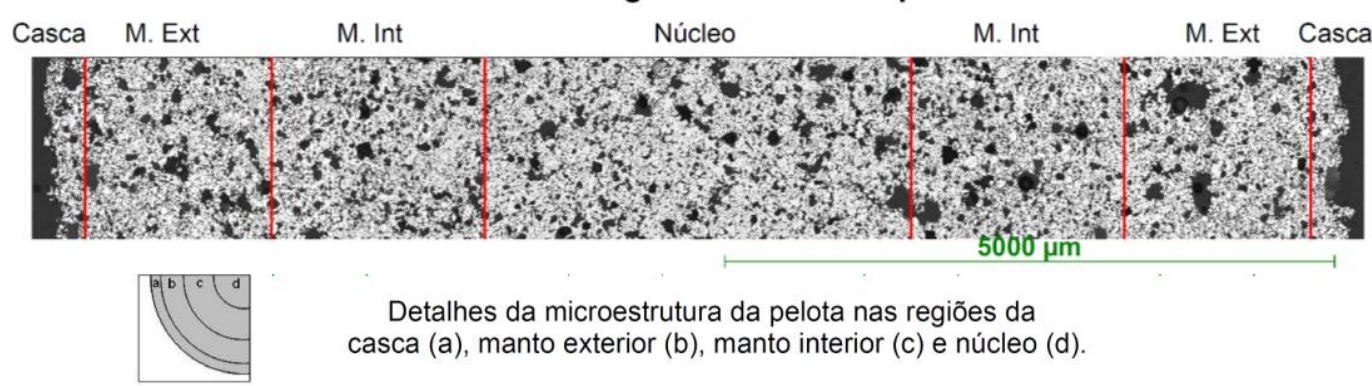

Detalhes da microestrutura da pelota nas regiões da casca (a), manto exterior (b), manto interior (c) e núcleo (d).

Figura 10. Mosaico diametral obtido em seção completa da pelota de minério de ferro.

O grau de evolução microestrutural refere-se as transformações ocorridas aos grãos originais de hematita, que se modificam sob efeito da temperatura e em contato com as fases líquidas que se formam durante a queima da pelota. Ocorre um ajuste morfológico entre os grãos e a matriz que pode promover aumento na resistência física da pelota [10]. O grau de evolução é classificado em cada região da pelota e é um indicativo da intensidade de queima (Figura 11).
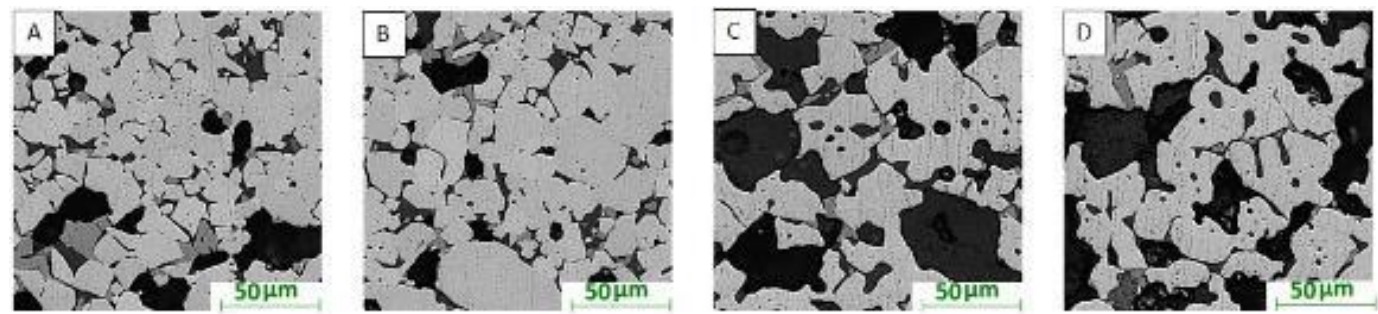

Figura 11. Detalhes da evolução microestrutural dos grãos. (A) casca, (B) manto externo, (C) manto interno, (D) núcleo 
A análise de determinação das fases e da porosidade também é realizada em cada região da pelota, e é feita a partir da segmentação dos histogramas. Na Figura 12 é mostrado um relatório que foi gerado automaticamente em formato .pdf na análise de pelotas.
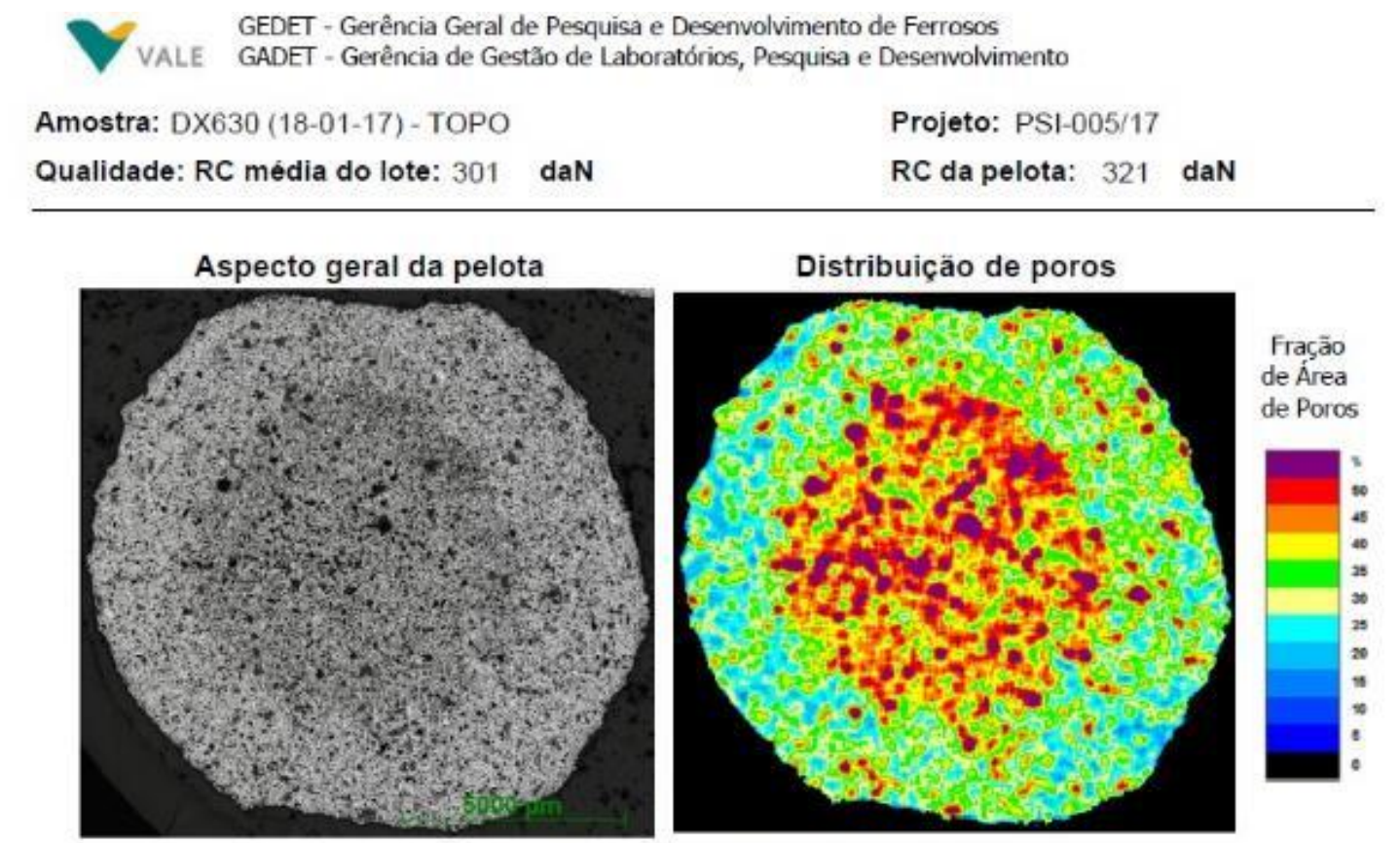

Análise da regiāo diametral da pelota
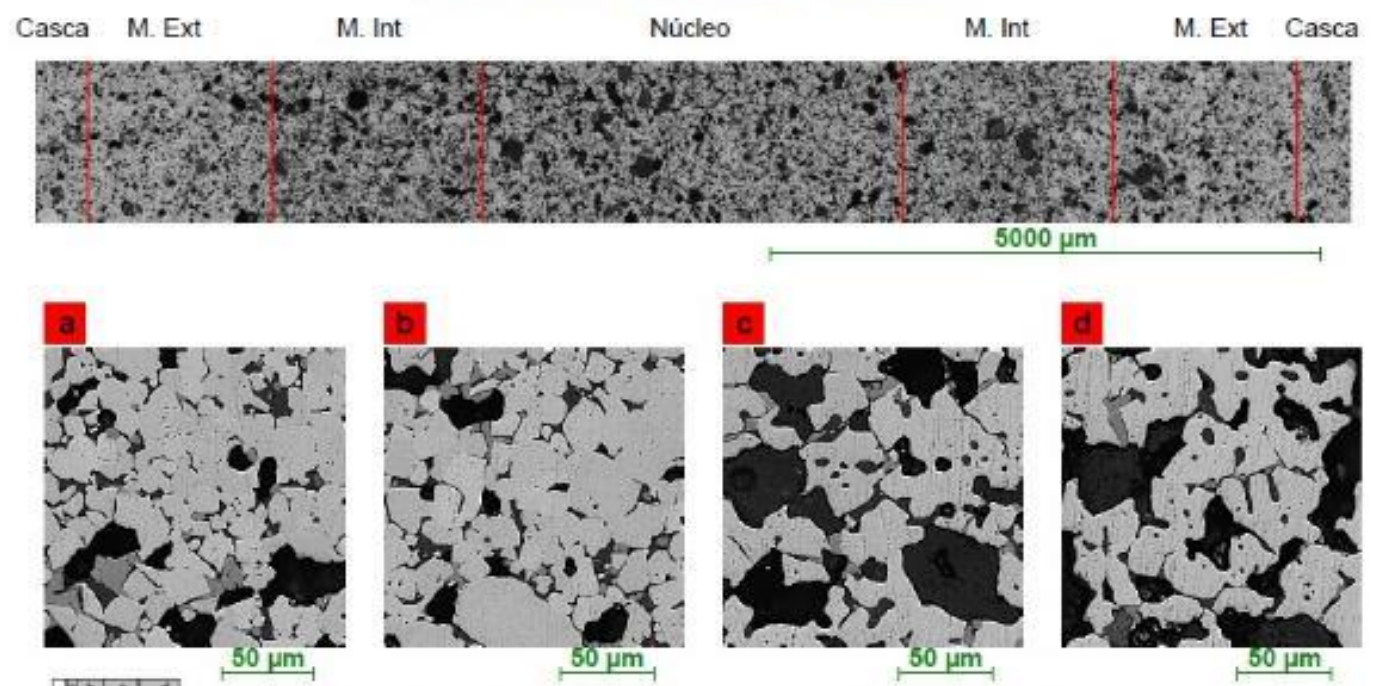

Detalhes da microestrutura da pelota nas regiões da casca (a), manto exterior (b), manto interior (c) e núcleo (d).

Fração de fase por região (\%)

\begin{tabular}{|c|c|c|c|c|c|c|c|}
\hline Regiāo & Poros[] & Escória[] & Ferrito[] & Magnetita[] & Hematita[] & Total[] & Maturaçảo \\
\hline Casca & 30.48 & 3.07 & 3.67 & 4.53 & 88.73 & 69.52 & C \\
\hline M.Ext & 33.33 & 3.26 & 4.11 & 5.77 & 86.87 & 66.67 & $\mathrm{C}$ \\
\hline M.Int & 43.60 & 4.12 & 4.52 & 8.09 & 83.26 & 56.41 & $\mathrm{D}$ \\
\hline Núcleo & 44.00 & 3.93 & 4.57 & 8.61 & 82.89 & 56.00 & $\mathrm{D}$ \\
\hline Média Tot & 39.80 & 3.73 & 4.35 & 7.32 & 84.59 & & \\
\hline
\end{tabular}

Figura 12. Exemplo de um relatório automático gerado após a execução da rotina de análise de pelota. 


\section{CONCLUSÃO}

Por meio da análise de imagens de microscopia óptica, podem ser determinadas diversas propriedades dos minérios de ferro e seus aglomerados de forma sistematizada. A substituição das análises convencionais por análises automáticas trouxe ganhos para os diversos setores envolvidos, a saber:

- Diminuição do tempo de análises;

- Aumento da reprodutividade;

- Aumento da representatividade;

- Método não exaustivo para os operadores;

- Eliminação da subjetividade da análise;

- Geração de relatórios automáticos.

O sistema de análise de imagens permite que os resultados sejam empregados tanto no desenvolvimento de novos produtos quanto no controle de processos, controle de qualidade e aplicação no cliente final. Apesar de serem muito importantes para o entendimento do comportamento desses materiais nas diversas etapas da cadeia mínero-metalúrgica, muitas dessas análises são pouco difundidas na indústria em função da dificuldade em executar as análises de microscopia óptica de forma "manual". A microscopia automática com análise de imagens possibilita a difusão desse conhecimento na indústria de forma rápida, segura e eficiente.

\section{REFERÊNCIAS}

1 Domingues, ALA. Automated Optical Microscopy with Image Analysis as a Tool for Characterization of Iron Ore and Agglomerates in Mining and Steel Industries. In: 26 Congresso Brasileiro de Microscopia 2017. Anais do 26 Congresso Brasileiro de Microscopia 2017. Buzios: SBMM.

2 Iglesias, JCA; Gomes, OFM.; Paciornik, S. Automatic recognition of hematite grains under polarized reflected light microscopy through image analysis, Minerals Engineering, 2012, 24:1264-1270.

3 Gomes, OFM.; Iglesias, JCA; Paciornik, S; Vieira, M. B. Classification of hematite types in iron ores through circularly polarized light microscopy and image analysis, Minerals Engineering, 2013, 52:191-197.

4 Iglesias, JCA . Automatic Characterization of Iron Ore through Image Processing and Analysis. In: 26 Congresso Brasileiro de Microscopia 2017. Anais do 26 Congresso Brasileiro de Microscopia 2017. Buzios: SBMM.

5 Gomes, OFM.; Iglesias, JCA; Paciornik, S.; Vieira, M. B. Morfometria e classificação automática de hematita em minérios de ferro. In: 14 Simpósio Brasileiro de Minério de Ferro, 2013. Anais do 14 Simpósio Brasileiro de Minério de Ferro. São Paulo: ABM. v. 1. p. $882-892$.

6 Gomes, OFM.; Iglesias, JCA; Paciornik, S.; Vieira, MB. Classification of Hematite Types in Iron Ores through Circularly Polarized Light Microscopy and Image Analysis. In: Process Mineralogy '12, 2012, Cidade do Cabo. Process Mineralogy '12. Falmouth: Minerals Engineering International, 2012. p. 1-14. 
7 Iglesias, JCA. Desenvolvimento de um Sistema de Microscopia Digital para Classificação Automática de Tipos de Hematita em Minério de Ferro. Tese de Doutorado. Rio de Janeiro: Pontifícia Universidade Católica do Rio de Janeiro, 2012.

8 Gomes, OFM.; Iglesias, JCA; Mauricio, MHP, Paciornik, S.; Vieira, MB. Caracterização automática de sínter de minério de ferro. In: 1 Simpósio Brasileiro de Minério de Ferro, 2013, Belo Horizonte. Anais do 1 Simpósio Brasileiro de Minério de Ferro. São Paulo: ABM, 2013. p. 893-901.

9 Wagner, DT, Rouco VR, Gomes, OFM, Paciornik, S, Vieira, MB. Caracterização de pelotas de minério de ferro por microscopia digital e análise de imagens. Tecnologia em Metalurgia Materiais e Mineração. 2009; 5(4) 215-218.

10 Augusto, KS. Identificação Automática do Grau de Maturação de Pelotas de Minério de Ferro. Dissertação de Mestrado. Rio de Janeiro: Pontifícia Universidade Católica do Rio de Janeiro, 2012. 